\title{
Quantum Simulation of Gauge Theories and Inflation
}

\author{
1. Self-Verifying Variational Quantum Simulation of the Lattice Schwinger \\ Model \\ Authors: C. Kokail et al. \\ arXiv:1810.03421
}

2. Probing quantum critical dynamics on a programmable Rydberg simulator Authors: Alexander Keesling et al.

Nature 568, 207 (2019)

\section{A Rapidly Expanding Bose-Einstein Condensate: An Expanding Universe in the Lab}

Authors: S. Eckel et al.

Phys. Rev. X, 8, 021021 (2018)

\section{Recommended with a Commentary by Steven M. Girvin, Yale Quantum Institute}

We have entered a new era of rapidly growing ability to precisely control and measure the complex states of quantum systems with ever larger Hilbert space dimensions. These capabilities are giving us new tools for studying novel quantum many-body systems. The simplest scenario is essentially that of an 'analog' quantum computer. In this case one has a system whose natural degrees of freedom and Hamiltonian match those of some other system one wishes to understand. An example of such a system is spin- $1 / 2$ fermionic atoms in an optical lattice. This system is a close approximation to the Hubbard model believed to describe the essential physics of quantum antiferromagnetic insulators and high-temperature superconductors. The cold atom system is naturally well-described by the Hubbard model and differs only in the relevant energy and temperature scales from the corresponding 'real' materials being simulated.

The advantage of such an analog quantum simulator is not simply that it contains the same physics, but that it allows the experimenter to make much more detailed measurements. Some of these measurements are unique to atomic physics and permit direct access to the many-body wave function, something that is generally not possible in condensed matter systems such as high-temperature superconductors. For example, a 'quantum gas microscope' can be used to take a series of snapshots of the spatial configurations of all the fermions in the quantum ground state. Another advantage of an analog quantum simulator 
is that typically one has at least some level of control over the Hamiltonian parameters. For example, in the case of an optical lattice, the experimenter can change the depth of the optical lattice potential by adjusting the laser intensity. This in turn adjusts the tunneling amplitude between sites since it depends sensitively on the barrier height set by the depth of the optical potential. Other atomic physics techniques (e.g. Feshbach resonances) can be used to adjust the interaction strength between the fermions.

With increasing levels of control it is possible to synthesize Hamiltonians that differ from the 'natural' Hamiltonian of the analog computer. For example periodic 'Floquet' modulation of some Hamiltonian terms can be used to create artificial gauge fields which vary from site to site and make neutral atoms in an optical lattice behave as if they were charged particles in a very strong magnetic field.

The ultimate limit of control is embodied in the universal quantum computer. Such a machine can be programmed to execute any possible unitary transformation on its own quantum state. Thus it can simulate time evolution under any possible Hamiltonian for its degrees of freedom. Using the fact that measurements cause partial state collapse, such a machine can even simulate any possible dissipative bath to which its degrees of freedom can be connected. It is thus a truly universal quantum device. It could for example use two-level qubits to simulate spin $S$ objects (not just $S=1 / 2$ ), hard- and soft-core bosons, as well as fermions. Given universal control, it could simulate a lattice fermion model in arbitrary spatial dimension $d$ using physical qubits laid out in 1,2 or 3 physical dimensions.

Kokail et al. use a programmable 20-qubit trapped-ion quantum simulator to create a variational approximation to the ground state of the lattice Schwinger model-a lattice gauge theory of $1+1-\mathrm{D}$ electrodynamics that happens to be exactly soluble. This is a hybrid simulator in which the quantum system is used as a 'co-processor' that is called upon by a classical processor to executes the calculation of the variational energy for a parameterized trial wave function. The classical processor then handles the variational optimization of parameters. The quantum co-processor is not an analog realization of the Schwinger Hamiltonian, but rather a digitally programmable system whose quantum gates create the desired variational state.

The optimal variational state generated by the finite-depth (up to depth 6 with up to 15 variational parameters) gate circuit has fidelity approaching 0.8 with the exact ground state for 20 spins (0.9 for 16 spins) and this number has been further improved since the preprint was posted (P. Zoller, private communication). One additional feature of this experiment is that the quality of the variational estimate for the ground state energy can be determined from the fluctuations in the measured energy. The energy variance approaches zero as the variational state approaches an energy eigenstate.

Keesling et al. use a different hardware platform consisting of a $1 \mathrm{D}$ array of $51{ }^{87} \mathrm{Rb}$ atoms to study the quantum Kibble-Zurek mechanism. Each atom acts as an effective two-level system consisting of the atomic ground state and a particular highly-excited Rydberg state. The collection of interacting qubits can be utilized to study the real-time non-equilibrium critical dynamics of several different models with $Z_{n}$ symmetry, the simplest of which is the transverse field Ising model with $Z_{2}$ symmetry. When the system is driven through the quantum phase transition at various finite rates (by varying tuning parameters in the Hamiltonian), the measured density of domain walls scales as a power of the rate, with the power-law exponent depending on the equilibrium correlation length exponent and the 
dynamical critical exponent of the model. Emerging technologies for realizing 2D Rydberg simulators will offer even greater quantum advantage because strongly correlated 2D systems can have greater entanglement than 1D systems and are thus less amenable to direct analysis on classical computers.

Eckel et al. offer an interesting analog quantum simulation of a 1D model for the nonequilibrium dynamics of cosmic inflation. In this experiment a circular ring of a Bose-Einstein condensate of radius $R(t)$ is expanded radially at a speed exceeding the speed of sound in the BEC. Phonon sound waves are red-shifted in frequency and stretched in wavelength as the expansion proceeds. Hints of 'Hubble friction' appear in the dynamics associated with an additional dissipative term in the equation of motion for the angular coordinate $\theta$ of a particle

$$
\ddot{\theta}=-2 \frac{\dot{R}}{R} \dot{\theta}+\ldots
$$

Hubble friction is crucial to the gravitational instability that caused galaxy formation in the early universe. Once a region of excess matter density forms, additional matter falls into the potential well region but is prevented from rolling up and out the other side by the Hubble friction associated with the expansion of the universe, thus causing small density fluctuations to grow.

These three papers are but a small sample of the rapidly developing experimental and theoretical activity in creation of ever more sophisticated quantum simulators. (A few additional references are listed below.) There is interesting quantum science and engineering in the building of these devices and much interesting physics to be explored with them once they are built.

\section{References}

[1] ' $U(1)$ Wilson lattice gauge theories in digital quantum simulators,' Christine Muschik et al., New J. Phys. 19, 103020 (2017).

[2] 'Quantum simulation of the Schwinger model: A study of feasibility,' Stefan Kühn, Phys. Rev. A 90, 042305 (2014).

[3] 'Real-time dynamics of lattice gauge theories with a few-qubit quantum computer,' Esteban A. Martinez et al., Nature 534, 5161 (2016).

[4] 'Quantum-classical computation of Schwinger model dynamics using quantum computers,' N. Klco et al., Phys. Rev. A 98, 032331 (2018). 